\title{
Study of a generalized logistic equation with nonlocal reaction term
}

Jianhua Zhou', Ge Gao and Baoqiang Yan²*

"Correspondence:

yanbqcn@aliyun.com

${ }^{2}$ School of Mathematical Sciences,

Shandong Normal University, Jinan,

P.R. China

Full list of author information is

available at the end of the article

\section{Abstract}

In this paper, we consider the generalized logistic equation with nonlocal reaction term

$$
-\Delta u=u\left(\lambda+b \int_{\Omega} u^{r} d x-f(u)\right) \quad \text { in } \Omega, \quad u>0 \quad \text { in } \Omega, \quad u=0 \quad \text { on } \partial \Omega .
$$

Using the bifurcation and sub-supersolution method, we obtain the non-existence, existence, and uniqueness of positive solutions for different parameters on the nonlocal terms. Our works about the nonlocal elliptic problem improve the results in the previous literature.

MSC: 35R09; 45K05; 35J60; 35J25

Keywords: Logistic equation; Nonlocal term; Bifurcation method; Sub-supersolution method; Existence

\section{Introduction}

In this paper, we consider the nonlocal elliptic boundary value problem

$$
\left\{\begin{array}{l}
-\Delta u=u\left(\lambda+b \int_{\Omega} u^{r} d x-f(u)\right), \quad \text { in } \Omega, \\
u>0, \quad \text { in } \Omega, \\
u=0, \quad \text { on } \partial \Omega .
\end{array}\right.
$$

Here $\Omega$ is a bounded domain in $\mathbb{R}^{N}, N \geq 2$, with $C^{2, \beta}$ boundary $\partial \Omega, \lambda, b \in \mathbb{R}, r>0, \beta \in$ $(0,1)$, and $f(u)$ is a polynomial denoted by

$$
f(u)=\sum_{i=1}^{n} a_{i} u^{k_{i}}, \quad a_{i}>0, i=1,2, \ldots, n(n \geq 1)
$$

where

all $k_{i}$ are integers with $1=k_{1}<k_{2}<\cdots<k_{n}$.

\section{Springer}


This type of problem was studied initially by Delgado et al. in [8], where they proposed the equation

$$
\left\{\begin{array}{l}
\frac{\partial u}{\partial t}-\Delta u=u\left(\lambda+b \int_{\Omega} u^{r} d x-u\right), \quad \text { in } \Omega \times(0,+\infty) \\
u(x, t)=0, \quad \text { in } \partial \Omega \times(0,+\infty), \\
u(x, 0)=u_{0}, \quad \text { on } \Omega
\end{array}\right.
$$

and the corresponding steady-state problem

$$
\left\{\begin{array}{l}
-\Delta u=u\left(\lambda+b \int_{\Omega} u^{r} d x-u\right), \quad \text { in } \Omega \\
u>0, \quad \text { in } \Omega, \\
u=0, \quad \text { on } \partial \Omega .
\end{array}\right.
$$

Here $u(x, t)$ represents the density of a species in time $t>0$ and at the point $x \in \Omega$, the habitat of the species that is surrounded by inhospitable areas, $\lambda$ is the growth rate of species, term $-f(u)$ describes the limiting effect of crowding in the population. In this paper the authors proved the existence of an unbounded continuum of positive solutions of (1.3), presented some non-existence results, and discussed the local and global behavior of the continuum.

The introduction of nonlocal terms in the equation and in the boundary conditions models a number of processes in different fields such as mathematical physics, mechanics of deformable solids, mathematical biology, and many others (see [1, 2, 4, 5, 10-12, 16, 20]).

Obviously, problem (1.1) is a generalization of problem (1.3). In this paper, we present some results on the existence of an unbounded continuum of positive solutions of (1.1), the local and global behavior of the continuum, and prove the non-existence of positive solutions also.

The paper is organized as follows. In Sect. 2 we give some lemmas which show the relationship among the solution, sub-solution, and super-solution and the relationship between the solution and the nonlinear term $f$ and prove the existence of an unbounded continuum of positive solutions of (1.1). Section 3 is devoted to proving the non-existence results and a priori bounds of positive solutions of (1.1). In Sect. 4 we presents some conditions for the existence of positive solutions for (1.1) and prove local and global behavior of the continuum of positive solutions of (1.1). Some ideas come from [13, 14].

Throughout our paper, we always suppose that (1.2) is true.

\section{Bifurcation results}

In order to discuss (1.1), we consider the following equation:

$$
\left\{\begin{array}{l}
-\Delta u=u(\lambda-f(u)), \quad \text { in } \Omega \\
u>0, \quad \text { in } \Omega \\
u=0, \quad \text { on } \partial \Omega
\end{array}\right.
$$

where $\lambda \in \mathbb{R}$. 
Denote by $\varphi_{1}$ an eigenfunction corresponding to the principle eigenvalue $\lambda_{1}$ of

$$
\left\{\begin{array}{l}
-\Delta u=\lambda u, \quad \text { in } \Omega \\
u=0, \quad \text { on } \partial \Omega .
\end{array}\right.
$$

From [9] and [15], $\varphi_{1}$ belongs to $C^{2, \beta}(\bar{\Omega}), \varphi_{1}>0$ in $\Omega$, and $\lambda_{1}>0$. Moreover, assume that $\left\|\varphi_{1}\right\|_{\infty}=1$.

Lemma 2.1 (See [3]) There exists a positive solution of (2.1) if and only if $\lambda>\lambda_{1}$. Moreover, if it exists, the solution is unique, and we denote it by $\theta_{\lambda}$. Furthermore, the following inequalities hold:

$$
f^{-1}\left(\lambda-\lambda_{1}\right) \varphi_{1} \leq \theta_{\lambda} \leq f^{-1}(\lambda)
$$

Lemma 2.2 Assume that $u$ is the unique positive solution to (2.1) for $\lambda>\lambda_{1}$. Then

$$
u=\left(\lambda-\lambda_{1}\right) m_{1}^{-1} \varphi_{1}+\left(\lambda-\lambda_{1}\right)^{2} m_{1}^{-2} U_{1}+O\left(\left|\lambda-\lambda_{1}\right|^{3}\right), \quad \text { as } \lambda \downarrow \lambda_{1},
$$

where

$$
m_{1}:=a_{1} \int_{\Omega} \varphi_{1}^{3} d x \neq 0
$$

and we have denoted by $\beta_{1}$ the unique solution of the following linear problem in $\Omega$ under the homogeneous Dirichlet boundary condition:

$$
\left(-\Delta-\lambda_{1}\right) \beta_{1}=m_{1} \varphi_{1}-a_{1} \varphi_{1}^{2}, \quad \int_{\Omega} \beta_{1} \varphi_{1} d x=0,
$$

and

$$
\begin{aligned}
& m_{2}:=2 a_{1} \int_{\Omega} \varphi_{1}^{2} \beta_{1} d x+a_{2} \int_{\Omega} \varphi_{1}^{4} d x-m_{1} \int_{\Omega} \beta_{1} \varphi_{1} d x, \quad \text { if } k_{2}=2, \\
& m_{2}:=2 a_{1} \int_{\Omega} \varphi_{1}^{2} \beta_{1} d x-m_{1} \int_{\Omega} \beta_{1} \varphi_{1} d x, \quad \text { if } k_{2}>2, \\
& U_{1}:=\beta_{1}-\frac{m_{2}}{m_{1}} \varphi_{1} .
\end{aligned}
$$

Lemma 4.3 in [9] proved the relationship between the solution $u$ of problem (2.1) when $f(u)=u$ and the first eigenfunction $\varphi_{1}$ of problem (2.2). Lemma 2.2 obtains a similar result for the case $f(u)=\sum_{i=1}^{n} a_{i} u^{k_{i}}$. Since the proof is the same as that in [9], we omit it.

From Lemma 2.2, we obtain the following corollary directly.

Corollary 2.1 Assume that $u$ is the unique positive solution to (2.1) for $\lambda>\lambda_{1}$. There exist two positive constants $\delta>0$ and $K>0$ such that

$$
u \leq K\left(\lambda-\lambda_{1}\right) \varphi_{1}, \quad \forall \lambda \in\left(\lambda_{1}, \lambda_{1}+\delta\right] .
$$


Lemma 2.3 Assume that $\theta_{\lambda}$ is the unique positive solution to (2.1) for $\lambda>\lambda_{1}$.

(1) If $\underline{u}>0$ is a strict sub-solution of (2.1), then $\underline{u} \leq \theta_{\lambda}$.

(2) If $\bar{u}>0$ is a strict super-solution of (2.1), then $\theta_{\lambda} \leq \bar{u}$.

Since $u^{-1} u(\lambda-f(u))=\lambda-f(u)$ is decreasing, it is easy to get the proof from Lemma 2.3 in [19], and we omit it.

We consider the Banach space $X:=C_{0}(\bar{\Omega})$, denote $B_{\rho}:=\left\{u \in X:\|u\|_{\infty}<\rho\right\}$. Define

$$
F(u)=u\left(\lambda+b \int_{\Omega} u_{+}^{r} d x-f(u)\right)
$$

and the map

$$
K_{\lambda}: X \rightarrow X, \quad K_{\lambda}(u)=u-(-\Delta)^{-1}(F(u))
$$

where $u_{+}=\max \{u(x), 0\}$ and $(-\Delta)^{-1}$ is the inverse of the operator $-\Delta$ under homogeneous Dirichlet boundary conditions. Agmon-Douglas-Nirenberg theorem, embedding theorem, and strong maximum theorem(see [17]) guarantee that $(-\Delta)^{-1}$ is positive and compact. It is clear that $u$ is a nonnegative solution of (1.1) if and only if $K_{\lambda} u=0$.

Now we give the main result of this section.

Theorem 2.1 The value $\lambda=\lambda_{1}$ is the only bifurcation point from the trivial solution for (1.1). Moreover, there exists a continuum $C_{0}$ of nonnegative solutions of (1.1) unbounded in $\mathbb{R} \times X$ emanating from $\left(\lambda_{1}, 0\right)$. Furthermore,

(i) if $b \leq 0$, the direction of bifurcation is supercritical.

(ii) Assume $b>0$.

(a) If $r<1$, the direction of bifurcation is subcritical.

(b) If $r>1$, then the direction of bifurcation is supercritical.

(c) Assume $r=1$, and denote

$$
b_{0}=\frac{a_{1} \int_{\Omega} \varphi_{1}^{3} d x}{\int_{\Omega} \varphi_{1} d x \int_{\Omega} \varphi_{1}^{2} d x} .
$$

If $b>b_{0}$ (resp. $\left.b<b_{0}\right)$, the direction of bifurcation is subcritical (resp. supercritical).

Recall that we say that the direction of bifurcation is subcritical (resp. supercritical) if there exists a neighborhood $V$ of $\left(\lambda_{1}, 0\right)$ such that for every solution $(\lambda, u) \in V$ satisfies $\lambda<\lambda_{1}$ (resp. $\lambda>\lambda_{1}$ ), see [8].

In order to prove this result, we use the Leray-Schauder degree of $K_{\lambda}$ on $B_{\rho}$ with respect to zero, denoted by $\operatorname{deg}\left(K_{\lambda}, B_{\rho}\right)$, and the index of the isolated zero of $K_{\lambda}$, denoted by $i\left(K_{\lambda}, u\right)$.

Lemma 2.4 If $\lambda<\lambda_{1}$, then $i\left(K_{\lambda}, 0\right)=1$.

Lemma 2.5 If $\lambda>\lambda_{1}$, then $i\left(K_{\lambda}, 0\right)=0$.

Since the proof is the same as that of Lemmas 2.3 and 2.4 in [8], we omit it. 
Proof of Theorem 2.1 From Lemmas 2.4 and 2.5 and bifurcation theorem (see [18]), the same proof as that of Theorem 2.2 in [8] guarantees the existence of an unbounded continuum $C_{0}$ of positive solutions of (1.1). Moreover, conclusion (i) is true.

We only give the proof of (ii).

(a) Assume now that $b>0$ and the existence of a sequence $\left(\lambda_{n}, u_{n}\right) \in C_{0}$ of positive solutions of (1.1) such that $\lambda_{n} \geq \lambda_{1}$ and $\left\|u_{n}\right\|_{\infty} \rightarrow 0$ as $n \rightarrow \infty$. By the property of $f$, there is $\delta_{1}>0$ such that

$$
f(u) \leq\left(a_{1}+1\right) u, \quad \forall u \in\left[0, \delta_{1}\right]
$$

Take $M>0$ such that

$$
\left(a_{1}+1\right)-b M \int_{\Omega} \varphi_{1} d x<0 .
$$

Since $r<1$, choose $n$ large enough such that $u_{n}^{r}>M u_{n}$, and then

$$
-\Delta u_{n}>u_{n}\left(\lambda_{n}+b M \int_{\Omega} u_{n} d x-f\left(u_{n}\right)\right)
$$

which implies that $u_{n}$ is a strict super-solution of the following system:

$$
\left\{\begin{array}{l}
-\Delta u=u\left(\lambda_{n}+b M \int_{\Omega} u_{n} d x-f(u)\right), \quad x \in \Omega, \\
\left.u\right|_{\partial \Omega}=0 .
\end{array}\right.
$$

Using Lemma 2.1, we get (2.7) has a unique positive solution $\theta_{n}$ and

$$
\theta_{n} \geq f^{-1}\left(\lambda_{n}+b M \int_{\Omega} u_{n} d x-\lambda_{1}\right) \varphi_{1}
$$

Lemma 2.3 implies that

$$
u_{n} \geq \theta_{n} \geq f^{-1}\left(\lambda_{n}+b M \int_{\Omega} u_{n} d x-\lambda_{1}\right) \varphi_{1}
$$

Integrating the above inequality yields that

$$
\int_{\Omega} u_{n} d x \geq f^{-1}\left(\lambda_{n}+b M \int_{\Omega} u_{n} d x-\lambda_{1}\right) \int_{\Omega} \varphi_{1} d x
$$

And then

$$
f\left(\frac{\int_{\Omega} u_{n} d x}{\int_{\Omega} \varphi_{1} d x}\right) \geq \lambda_{n}+b M \int_{\Omega} u_{n} d x-\lambda_{1}
$$

Using (2.5), one has

$$
\left(a_{1}+1\right)\left(\int_{\Omega} \varphi_{1} d x\right)^{-1} \int_{\Omega} u_{n} d x \geq f\left(\frac{\int_{\Omega} u_{n} d x}{\int_{\Omega} \varphi_{1} d x}\right)
$$


for $n$ large enough. And then

$$
\left(a_{1}+1\right)\left(\int_{\Omega} \varphi_{1} d x\right)^{-1} \int_{\Omega} u_{n} d x \geq \lambda_{n}+b M \int_{\Omega} u_{n} d x-\lambda_{1}
$$

which together with (2.6) implies

$$
0>\left(\left(a_{1}+1\right)\left(\int_{\Omega} \varphi_{1} d x\right)^{-1}-b M\right) \int_{\Omega} u_{n} d x \geq \lambda_{n}-\lambda_{1}
$$

an absurdum.

(b) Assume now that $b>0, r>1$ and the existence of a sequence $\left(\lambda_{n}, u_{n}\right) \in C_{0}$ of positive solutions of (1.1) such that $\lambda_{n} \leq \lambda_{1}$ and $\left\|u_{n}\right\|_{\infty} \rightarrow 0$ as $n \rightarrow \infty$. Without loss of generality, assume that $\left\|u_{n}\right\|_{\infty} \leq \delta$ defined in Corollary 2.1. Take $\varepsilon>0$ such that

$$
1-b K \varepsilon|\Omega|>0
$$

where $K$ is defined in Corollary 2.1.

For $n$ large we have $u_{n}^{r}<\varepsilon u_{n}$, and then

$$
-\Delta u_{n}<u_{n}\left(\lambda_{n}+b \varepsilon \int_{\Omega} u_{n} d x-f\left(u_{n}\right)\right)
$$

which implies that $u_{n}$ is a strict sub-solution of the following problem:

$$
\left\{\begin{array}{l}
-\Delta v=v\left(\lambda_{n}+b \varepsilon \int_{\Omega} u_{n} d x-f(v)\right), \quad x \in \Omega \\
\left.v\right|_{\partial \Omega}=0 .
\end{array}\right.
$$

By Lemma 2.1, we get (2.9) has a unique positive solution $\theta_{n}$. Moreover, from Corollary 2.1, we have

$$
\theta_{n} \leq K\left(\lambda_{n}+b \varepsilon \int_{\Omega} u_{n} d x-\lambda_{1}\right) \varphi_{1} \leq K\left(\lambda_{n}+b \varepsilon \int_{\Omega} u_{n} d x-\lambda_{1}\right)
$$

for $n$ large enough. By Lemma 2.3, we have

$$
u_{n} \leq \theta_{n} \leq K\left(\lambda_{n}+b \varepsilon \int_{\Omega} u_{n} d x-\lambda_{1}\right) \varphi_{1} \leq K\left(\lambda_{n}+b \varepsilon \int_{\Omega} u_{n} d x-\lambda_{1}\right) .
$$

Integrating the above inequality yields that

$$
\int_{\Omega} u_{n} d x \leq K\left(\lambda_{n}+b \varepsilon \int_{\Omega} u_{n} d x-\lambda_{1}\right)|\Omega|
$$

which together with (2.8) implies that

$$
0<(1-K b \varepsilon|\Omega|) \int_{\Omega} u_{n} d x \leq K\left(\lambda_{n}-\lambda_{1}\right)|\Omega|
$$

an absurdum.

(c) Assume that $b>0$ and $r=1$. In this case, we apply the Crandall-Rabinowitz theorem (see [7]). Then there exist $\varepsilon>0$ and two regular functions $\lambda(s), u(s), s \in(-\varepsilon, \varepsilon)$, such that 
in a neighborhood of $\left(\lambda_{1}, 0\right)$, the positive solutions are $u(s), s \in(0, \varepsilon)$. We can write

$$
u(s)=s \varphi_{1}+s^{2} \varphi_{2}+o\left(s^{2}\right)
$$

and

$$
\lambda(s)=\lambda_{1}+s \lambda_{2}+o(s)
$$

where $\lambda_{2} \in \mathbb{R}, \varphi_{2} \in C^{2}(\bar{\Omega})$. It is evident that the sign of $\lambda_{2}$ determines the bifurcation direction. Substituting these expansions into (1.1) and identifying the terms of order one in $s$ yield

$$
-\Delta \varphi_{2}-\lambda_{1} \varphi_{2}=\lambda_{2} \varphi_{1}-a_{1} \varphi_{1}^{2}+b \varphi_{1} \int_{\Omega} \varphi_{1} d x
$$

Multiplying by $\varphi_{1}$ and integrating in $\Omega$, we conclude that

$$
\lambda_{2}=\frac{a_{1} \int_{\Omega} \varphi_{1}^{3} d x-b \int_{\Omega} \varphi_{1}^{2} d x \int_{\Omega} \varphi_{1} d x}{\int_{\Omega} \varphi_{1}^{2} d x} .
$$

This finishes the proof.

\section{A priori bounds and non-existence results of (1.1)}

In this section, we obtain a priori bounds of the solutions for $b>0$ as well as non-existence results of (1.1).

Theorem 3.1 Assume that $b>0$ and $r<1$. Let $u_{\lambda}$ be a positive solution of (1.1) such that $\lambda \in K \subset \mathbb{R}$ a compact set. Then there exists a constant $L_{0}>0$ such that

$$
\left\|u_{\lambda}\right\|_{\infty} \leq L_{0}
$$

for a constant independent of $\lambda \in K$. Moreover, there exists a constant $L_{1}$ such that, if

$$
\lambda<L_{1}
$$

(1.1) does not possess any positive solution.

Proof Since $K$ is compact, there is a positive constant $k_{0}>0$ such that

$$
\lambda \leq k_{0}, \quad \forall \lambda \in K
$$

Moreover, since $u_{\lambda}$ is a positive solution of (1.1), we have, using Lemma 2.1 and Hölder's inequality, that

$$
\begin{aligned}
u_{\lambda} & \leq f^{-1}\left(\lambda+b \int_{\Omega} u_{\lambda}^{r} d x\right) \\
& \leq f^{-1}\left(\lambda+b|\Omega|^{1-r}\left(\int_{\Omega} u_{\lambda} d x\right)^{r}\right) \\
& \leq f^{-1}\left(k_{0}+b|\Omega|^{1-r}\left(\int_{\Omega} u_{\lambda} d x\right)^{r}\right), \quad x \in \Omega .
\end{aligned}
$$


Step 1. We show that there exists $c_{1}>0$ such that

$$
\int_{\Omega}\left|u_{\lambda}\right| d x \leq c_{1}
$$

In fact, suppose to the contrary that there exists $\left\{u_{\lambda_{n}}\right\}$ such that

$$
\int_{\Omega} u_{\lambda_{n}} d x \rightarrow+\infty, \quad n \rightarrow+\infty
$$

Replacing $\lambda$ in (3.1) by $\lambda_{n}$, one has

$$
u_{\lambda_{n}} \leq f^{-1}\left(k_{0}+b\left(\int_{\Omega} u_{\lambda_{n}} d x\right)^{r}|\Omega|^{1-r}\right) .
$$

Integrating inequality (3.3) in $\Omega$ yields that

$$
\int_{\Omega} u_{\lambda_{n}} d x \leq f^{-1}\left(k_{0}+b|\Omega|^{1-r}\left(\int_{\Omega} u_{\lambda_{n}} d x\right)^{r}\right)|\Omega|,
$$

i.e.,

$$
f\left(|\Omega|^{-1} \int_{\Omega} u_{\lambda_{n}} d x\right) \leq \lambda_{n}+b|\Omega|^{1-r}\left(\int_{\Omega} u_{\lambda_{n}} d x\right)^{r} .
$$

By the property of $f(u)$, one has

$$
f(u) \geq a_{1} u,
$$

which together with (3.4) implies that

$$
a_{1}|\Omega|^{-1} \int_{\Omega} u_{\lambda_{n}} d x \leq k_{0}+b|\Omega|^{1-r}\left(\int_{\Omega}\left|u_{\lambda_{n}}\right| d x\right)^{r}
$$

for $n$ large enough. This is a contradiction because $r<1$.

Step 2. We show that there exists a constant $L_{0}>0$ such that

$$
\left\|u_{\lambda}\right\|_{\infty} \leq L_{0}, \quad \forall \lambda \in K
$$

Since Step 1 holds, (3.1) guarantees that

$$
u_{\lambda} \leq f^{-1}\left(\lambda+b\left(\int_{\Omega} u_{\lambda} d x\right)^{r}|\Omega|^{1-r}\right) \leq f^{-1}\left(k_{0}+b c_{1}^{r}|\Omega|^{1-r}\right) .
$$

Let $L_{0}:=f^{-1}\left(k_{0}+b c_{1}^{r}|\Omega|^{1-r}\right)$. We conclude

$$
\left\|u_{\lambda}\right\|_{\infty} \leq L_{0}, \quad \forall \lambda \in K
$$

Step 3. We show that there exists a constant $L_{1}$ such that, if

$$
\lambda<L_{1} \text {, }
$$

(1.1) does not possess any positive solution. 
Now define a function

$$
g(s):=f\left(|\Omega|^{-1} s\right)-b|\Omega|^{1-r} s^{r}, \quad s \in[0,+\infty)
$$

From the property of $f$ and $0<r<1$, one has

$$
\lim _{s \rightarrow+\infty} \frac{f\left(|\Omega|^{-1} s\right)}{s^{r}}=+\infty
$$

which together with $g(0)=0$ implies that there is $s_{0} \geq 0$ such that

$$
\min _{s \in[0,+\infty)} g(s)=g\left(s_{0}\right)
$$

Let

$$
L_{1}:=g\left(s_{0}\right)
$$

Assume that $u_{\lambda}$ is a positive solution to (1.1) for $\lambda \in \mathbb{R}$. From (3.4), we have

$$
f\left(|\Omega|^{-1} \int_{\Omega} u_{\lambda} d x\right)-b\left(\int_{\Omega} u_{\lambda} d x\right)^{r}|\Omega|^{1-r} \leq \lambda
$$

which means that

$$
\lambda \geq L_{1}
$$

Consequently, (1.1) has no positive solution if $\lambda<L_{1}$.

The proof is complete.

Theorem 3.2 Assume that $b>0, k_{n}<r$, where $k_{n}$ is the index of the last term of polynomial $f$. Let $u_{\lambda}$ be a positive solution of (1.1) such that $\lambda \in K \subset \mathbb{R}$ a compact set. Then there exists a constant $L_{0}$ such that

$$
\left\|u_{\lambda}\right\|_{\infty} \leq L_{0}
$$

for a constant independent of $\lambda \in K$. Moreover, there exists a constant $L_{1}$ such that

$$
\lim _{b \rightarrow+\infty} L_{1}=\lambda_{1}
$$

and if

$$
\lambda>L_{1},
$$

(1.1) does not possess any positive solution.

Proof Since $K$ is compact, there is $k_{1}$ such that

$$
k_{1} \leq \lambda, \quad \forall \lambda \in K
$$


Moreover, using now the lower bound in Lemma 2.1, we get that

$$
\begin{aligned}
f^{-1}\left(k_{1}+b \int_{\Omega} u_{\lambda}^{r} d x-\lambda_{1}\right) \varphi_{1} & \leq f^{-1}\left(\lambda+b \int_{\Omega} u_{\lambda}^{r} d x-\lambda_{1}\right) \varphi_{1} \\
& \leq u_{\lambda} \leq f^{-1}\left(\lambda+b \int_{\Omega} u_{\lambda}^{r} d x\right), \quad x \in \Omega
\end{aligned}
$$

Step 1 . We show that there exists a constant $c_{1}>0$ such that

$$
\int_{\Omega} u_{\lambda}^{r} d x \leq c_{1}, \quad \forall \lambda \in K
$$

In fact, suppose to the contrary that there exists $\left\{\lambda_{n}\right\} \subseteq K$ such that

$$
\int_{\Omega} u_{\lambda_{n}}^{r} d x \rightarrow+\infty, \quad \text { as } n \rightarrow+\infty
$$

By the property of $f(u)$, there is $M>0$ such that

$$
f(u) \leq M u^{k_{n}}
$$

for $u$ large enough. Integrating (3.5) in $\Omega$ yields that

$$
f^{-1}\left(\lambda_{n}+b \int_{\Omega} u_{\lambda_{n}}^{r} d x-\lambda_{1}\right) \int_{\Omega} \varphi_{1} d x \leq \int_{\Omega} u_{\lambda_{n}} d x
$$

that is, by Hölder's inequality and (3.6)

$$
\begin{aligned}
\lambda_{n}+b \int_{\Omega} u_{\lambda_{n}}^{r} d x-\lambda_{1} & \leq f\left(\frac{\int_{\Omega} u_{\lambda_{n}} d x}{\int_{\Omega} \varphi_{1} d x}\right) \\
& \leq M\left(\frac{\int_{\Omega} u_{\lambda_{n}} d x}{\int_{\Omega} \varphi_{1} d x}\right)^{k_{n}} \\
& \leq M\left(\int_{\Omega} \varphi_{1} d x\right)^{-k_{n}}\left(\int_{\Omega} u_{\lambda_{n}}^{r} d x\right)^{\frac{k_{n}}{r}}|\Omega|^{\frac{r-1}{r}}
\end{aligned}
$$

for $n$ large enough.

This is a contradiction to $k_{n}<r$.

Step 2. We show that there exists a constant $L_{0}>0$ such that

$$
\left\|u_{\lambda}\right\|_{\infty} \leq L_{0}, \quad \forall \lambda \in K
$$

Since $K$ is compact, there exists $k_{0}$ such that

$$
\lambda \leq k_{0}, \quad \forall \lambda \in K
$$

From (3.5) and Step 1, one has

$$
u_{\lambda} \leq f^{-1}\left(\lambda+b \int_{\Omega} u_{\lambda}^{r} d x\right) \leq f^{-1}\left(k_{0}+b c_{1}\right)
$$


Let

$$
L_{0}:=f^{-1}\left(k_{0}+b c_{1}\right)
$$

Then $L_{0}$ satisfies Step 2.

Step 3. We show that there exists a constant $L_{1}$ such that, if

$$
\lambda>L_{1},
$$

(1.1) does not possess any positive solution.

Now define

$$
g_{1}(s):=f\left(\frac{s^{\frac{1}{r}}}{\left(\int_{\Omega} \varphi_{1}^{r} d x\right)^{\frac{1}{r}}}\right)-b s+\lambda_{1} .
$$

Since $k_{n}<r$, one has

$$
\lim _{s \rightarrow+\infty} g_{1}(s)=-\infty,
$$

which together with $g_{1}(0)=0$ implies that there exists $s_{1} \geq 0$ such that

$$
\max _{s \in[0,+\infty)} g_{1}(s)=g_{1}\left(s_{1}\right) .
$$

Let

$$
L_{1}:=g_{1}\left(s_{1}\right)
$$

Assume that $u_{\lambda}$ is a positive solution to (1.1) for $\lambda \in \mathbb{R}$. Integrating (3.5) in $\Omega$ yields that

$$
\left(f^{-1}\left(\lambda+b \int_{\Omega} u_{\lambda}^{r} d x-\lambda_{1}\right)\right)^{r} \int_{\Omega} \varphi_{1}^{r} d x \leq \int_{\Omega} u_{\lambda}^{r} d x,
$$

i.e.,

$$
f^{-1}\left(\lambda+b \int_{\Omega} u_{\lambda}^{r} d x-\lambda_{1}\right) \leq\left(\frac{\int_{\Omega} u_{\lambda}^{r} d x}{\int_{\Omega} \varphi_{1}^{r} d x}\right)^{\frac{1}{r}} .
$$

And then

$$
\lambda+b \int_{\Omega} u_{\lambda}^{r} d x-\lambda_{1} \leq f\left(\left(\frac{\int_{\Omega} u_{\lambda}^{r} d x}{\int_{\Omega} \varphi_{1}^{r} d x}\right)^{\frac{1}{r}}\right)
$$

that is,

$$
\lambda \leq-b \int_{\Omega} u_{\lambda}^{r} d x+f\left(\left(\frac{\int_{\Omega} u_{\lambda}^{r} d x}{\int_{\Omega} \varphi_{1}^{r} d x}\right)^{\frac{1}{r}}\right)+\lambda_{1} .
$$


Hence

$$
\lambda \leq L_{1}
$$

Consequently, Step 3 is true.

Moreover, we consider

$$
h(s):=A s^{q}-\frac{b}{n} s+\frac{\lambda_{1}}{n}, \quad A>0,0<q<1 .
$$

It is easy to prove that $h(s)$ gets its maximum at $s=\left(\frac{b}{n A q}\right)^{\frac{1}{1-q}}$ and

$$
\max _{s \in[0,+\infty)} h(s)=A\left(\frac{b}{n A q}\right)^{\frac{q}{1-q}}-\frac{b}{n}\left(\frac{b}{n A q}\right)^{\frac{1}{1-q}}+\frac{\lambda_{1}}{n} .
$$

Let

$$
h_{i}(s):=a_{i} s^{\frac{k_{i}}{r}}-\frac{b}{n} s+\frac{\lambda_{1}}{n}, \quad i=1,2, \ldots
$$

Obviously, we have

$$
\max _{s \in[0,+\infty)} h_{i}(s)=a_{i}\left(\frac{b}{n A \frac{k_{i}}{r}}\right)^{\frac{\frac{k_{i}}{r}}{1-\frac{k_{i}}{r}}}-\frac{b}{n}\left(\frac{b}{n A \frac{k_{i}}{r}}\right)^{\frac{1}{1-\frac{k_{i}}{r}}}+\frac{\lambda_{1}}{n} .
$$

Since $0<\frac{k_{i}}{r}<1, i=1,2, \ldots, n$, one has that

$$
\lim _{b \rightarrow+\infty} \max _{s \in[0,+\infty)} h_{i}(s)=\frac{\lambda_{1}}{n}, \quad i=1,2,3, \ldots, n .
$$

By the definition $g_{1}$ in (3.7), we have

$$
g_{1}(s)=\sum_{i=1}^{n} h_{i}(s)
$$

which implies that

$$
\lim _{b \rightarrow+\infty} L_{1}=\lim _{b \rightarrow+\infty} \max _{s \in[0,+\infty)} g_{1}(s) \leq \lim _{b \rightarrow+\infty} \sum_{i=1}^{n} \max _{s \in[0,+\infty)} h_{i}(s)=\lambda_{1} .
$$

On the other hand, since $L_{1}>\lambda_{1}$, we have

$$
\lim _{b \rightarrow+\infty} L_{1}=\lambda_{1}
$$

The proof is complete.

Theorem 3.3 Assume that $b>0, r=1$. Let $u_{\lambda}$ be a positive solution of (1.1) such that $\lambda \in K \subset \mathbb{R}$ a compact set. 
(1) If $k_{n}=1=r$ and $b<1 /|\Omega|$ or $b \int_{\Omega} \varphi_{1} d x>1$, then there exist a priori bounds of the solution of (1.1). Moreover, if $b<1 /|\Omega|$ and $\lambda \leq 0$ or $b \int_{\Omega} \varphi_{1} d x>1$ and $\lambda \geq \lambda_{1}$, then (1.1) does not possess a positive solution.

(2) If $k_{n}>1=r$, then there exists a constant $L_{0}>0$ such that

$$
\left\|u_{\lambda}\right\|_{\infty} \leq L_{0}
$$

for a constant independent of $\lambda$. Moreover, there exists a constant $L_{1}$ such that, if

$$
\lambda<L_{1}
$$

(1.1) does not possess any positive solution.

Conclusion (i) is Proposition 3.1 in [8] and the proof of (ii) is similar to that in Theorem 3.1, and we omit it.

\section{Existence and uniqueness results}

In this section, first we introduce the method of sub-supersolution to some nonlocal elliptic problems.

Consider a continuous operator $B: L^{\infty}(\Omega) \rightarrow \mathbb{R}$ and $f: \Omega \times \mathbb{R}^{2} \rightarrow \mathbb{R}$ a continuous function and the general problem

$$
\left\{\begin{array}{l}
-\Delta u=f(x, u, B(u)), \quad \text { in } \Omega \\
u=0, \quad \text { on } \partial \Omega,
\end{array}\right.
$$

where $\Omega$ is a bounded domain in $\mathbb{R}^{N}, N \geq 2$, with $C^{2, \beta}$ boundary $\partial \Omega$.

Definition 4.1 (See [6]) We say that the pair $(\underline{u}, \bar{u})$ with $\underline{u}, \bar{u} \in H^{1}(\Omega) \cap L^{\infty}(\Omega)$ is a pair of sub-supersolutions of (4.1) if

(a) $\underline{u} \leq \bar{u}$ in $\Omega$ and $\underline{u} \leq 0 \leq \bar{u}$ on $\partial \Omega$;

(b) $-\Delta \underline{u}-f(x, \underline{u}, B(u)) \leq 0 \leq-\Delta \bar{u}-f(x, \bar{u}, B(u))$ in the weak sense for all $u \in[\underline{u}, \bar{u}]$.

Lemma 4.1 (See [6]) Assume that there exists a pair of sub-supersolutions of (4.1). Then there exists a solution $u \in H^{1}(\Omega) \cap L^{\infty}(\Omega)$ of $(4.1)$ such that $u \in[\underline{u}, \bar{u}]$.

Now we give the main theorems.

Theorem 4.1 Assume that $b<0$. Then (1.1) has a positive solution if and only if $\lambda>\lambda_{1}$. Moreover, if there exists the unique positive solution, denoted by $u_{\lambda, b}$, then

$$
\lim _{b \rightarrow-\infty}\left\|u_{\lambda, b}\right\|_{\infty}=0
$$

Proof By Theorem 2.1 we know the existence of an unbounded continuum $C_{0}$ of positive solutions bifurcating from the trivial solution at $\lambda=\lambda_{1}$. Assume that $\left(\lambda, u_{\lambda}\right) \in C_{0}$. Now we show that $\lambda>\lambda_{1}$. 
In fact, if $\lambda \leq \lambda_{1}$, then

$$
-\Delta u_{\lambda} \leq u_{\lambda}\left(\lambda-f\left(u_{\lambda}\right)\right)
$$

which implies that $u_{\lambda}$ is a sub-solution (2.1).

Choose a constant $K$ large enough such that

$$
\lambda<f(K) \text { and } K>\max _{x \in \bar{\Omega}} u_{\lambda} .
$$

Obviously, $\left(u_{\lambda}, K\right)$ is a pair of sub-supersolutions to (2.1). Then (2.1) has a positive solution for $\lambda \leq \lambda_{1}$. This is a contradiction to Lemma 2.1.

We know that positive solutions do not exist for $\lambda \leq \lambda_{1}$, hence we conclude that if $(\lambda, u) \in$ $C_{0}$, we have $\lambda>\lambda_{1}$.

Moreover, if $(\lambda, u) \in C_{0}$, since $b<0$, we have

$$
-\Delta u_{\lambda}<u_{\lambda}\left(\lambda-f\left(u_{\lambda}\right)\right)
$$

and Lemma 2.3 implies that $u_{\lambda} \leq \theta_{\lambda}$, where $\theta_{\lambda}$ is a solution to (2.1). Lemma 2.1 guarantees that (2.1) has a unique positive solution $\theta_{\lambda}$ for all $\lambda>\lambda_{1}$, which together with the unboundedness of $C_{0}$ implies that (1.1) has at least one positive solution $u_{\lambda, b}$ for all $\lambda>\lambda_{1}$.

We show now the uniqueness.

Assume that there exist two positive solutions $u \neq v$ for $b<0$. If $\int_{\Omega} u^{r} d x=\int_{\Omega} v^{r} d x, u$ and $v$ satisfy

$$
\left\{\begin{array}{l}
-\Delta u=u(\lambda+k-f(u)), \quad \text { in } \Omega, \\
u=0, \quad \text { on } \partial \Omega,
\end{array}\right.
$$

where $k=b \int_{\Omega} u^{r} d x=b \int_{\Omega} v^{r} d x<0$. This is a contradiction to Lemma 2.1.

So, assume that for instance

$$
\int_{\Omega} u^{r} d x<\int_{\Omega} v^{r} d x
$$

then

$$
-\Delta u=u\left(\lambda+b \int_{\Omega} u^{r} d x-f(u)\right)>u\left(\lambda+b \int_{\Omega} v^{r} d x-f(u)\right)
$$

and then by Lemma 2.3 we get $u>v$, an absurdum.

On the other hand, we have that

$$
u_{\lambda, b} \leq f^{-1}\left(\lambda+b \int_{\Omega} u_{\lambda, b}^{r} d x\right)
$$

and then $f\left(u_{\lambda, b}\right)<\lambda$. So, as $b \rightarrow-\infty$, we get

$$
\int_{\Omega} u_{\lambda, b}^{r} d x \rightarrow 0
$$


Moreover, Lemma 2.1 implies

$$
f^{-1}\left(\lambda+b \int_{\Omega} u_{\lambda, b}^{r} d x-\lambda_{1}\right) \varphi_{1} \leq u_{\lambda, b}
$$

and

$$
\lambda+b \int_{\Omega} u_{\lambda, b}^{r} d x-\lambda_{1}>0
$$

we conclude that

$$
b \int_{\Omega} u_{\lambda, b}^{r} d x \rightarrow \lambda_{1}-\lambda
$$

This implies that $\left\|u_{\lambda, b}\right\|_{\infty} \rightarrow 0$.

Theorem 4.2 Assume that $b>0,0<r<1$. Then there exists $\lambda_{*}<\lambda_{1}$ such that (1.1) possesses at least a positive solution if and only if $\lambda \geq \lambda_{*}$. Moreover,

$$
\lim _{b \rightarrow 0^{+}} \lambda_{*}(b)=\lambda_{1} \quad \text { and } \quad \lim _{b \rightarrow+\infty} \lambda_{*}(b)=-\infty
$$

Proof Define

$$
\lambda_{*}=\inf \{\lambda \in \mathbb{R}:(1.1) \text { possesses at least a positive solution }\} .
$$

We know by Theorems 2.1 and 3.1 that $-\infty<\lambda_{*}<\lambda_{1}$.

Step 1. We show that (1.1) has at least one positive solution for all $\lambda>\lambda_{*}$.

Take $\lambda>\lambda_{*}$, then there exists $\mu \in\left[\lambda_{*}, \lambda\right)$ such that (1.1) possesses at least a positive solution, denoted by $u_{\mu}$. Choose $K$ large enough such that

$$
f(K)-b K^{r}|\Omega|>\lambda \quad \text { and } \quad K>\max _{x \in \bar{\Omega}} u_{\mu}(x)
$$

Let $(\underline{u}, \bar{u})=\left(u_{\mu}, K\right)$. Since $u_{\mu}$ is a positive solution of (1.1) and (4.2) is true, we have

(a) $\underline{u}=u_{\mu}<K=\bar{u}$ in $\Omega$ and $\underline{u}=u_{\mu}=0<K=\bar{u}$ on $\partial \Omega$;

(b)

$$
\begin{aligned}
& -\Delta \underline{u}-\underline{u}\left(\lambda+b \int_{\Omega} u^{r} d x-f(\underline{u})\right) \\
& =u_{\mu}\left(\mu+b \int_{\Omega} u_{\mu}^{r} d x-f\left(u_{\mu}\right)\right)-u_{\mu}\left(\lambda+b \int_{\Omega} u^{r} d x-f\left(u_{\mu}\right)\right) \\
& =b u_{\mu} \int_{\Omega}\left(u_{\mu}^{r}-u^{r}\right) d x+(\mu-\lambda) u_{\lambda} \\
& \quad \leq 0, \quad x \in \Omega, \forall u \in[\underline{u}, \bar{u}]
\end{aligned}
$$


and

$$
\begin{aligned}
-\Delta \bar{u}-\bar{u}\left(\lambda+b \int_{\Omega} u^{r} d x-f(\bar{u})\right) & =-0-K\left(\lambda+b \int_{\Omega} u^{r} d x-f(K)\right) \\
& =K\left(-\lambda-b \int_{\Omega} u^{r} d x+f(K)\right) \\
& \geq K\left(-\lambda-b K|\Omega|^{r}+f(K)\right) \\
& >0, \quad x \in \Omega, \forall u \in[\bar{u}, \bar{u}],
\end{aligned}
$$

which implies that $(\underline{u}, \bar{u})$ is a pair of sub-supersolutions to (1.1). Theorem 4.2 guarantees that (1.1) has at least one positive solution for all $\lambda>\lambda_{*}$.

Step 2. We show that, for $\lambda=\lambda_{*},(1.1)$ has a positive solution.

By the definition of $\lambda_{*}$, there exists $\left\{\lambda_{n}\right\}$ such that $\lambda_{n} \geq \lambda_{*}$ and $\lambda_{n} \rightarrow \lambda_{*}$. Thanks to the bounds of Theorem 3.1, we have that $u_{n} \rightarrow u_{*} \geq 0, u_{*}$ is a solution for $\lambda=\lambda_{*}$. Since $\lambda_{*}<\lambda_{1}$ and $\lambda_{1}$ is the unique bifurcation point from the trivial solution, we conclude that $u_{*}>0$.

Step 3. We show that

$$
\lim _{b \rightarrow 0^{+}} \lambda_{*}(b)=\lambda_{1} \quad \text { and } \quad \lim _{b \rightarrow+\infty} \lambda_{*}(b)=-\infty
$$

Since $u$ is bounded and

$$
\lambda+b \int_{\Omega} u^{r} d x>\lambda_{1}
$$

and then taking $b \rightarrow 0$, we have that $\lambda \geq \lambda_{1}$, that is,

$$
\lim _{b \rightarrow 0^{+}} \lambda_{*}(b)=\lambda_{1}
$$

Now we prove

$$
\lim _{b \rightarrow+\infty} \lambda_{*}(b)=-\infty
$$

It suffices to show that, for any $\lambda<\lambda_{1}$, there exists $b>0$ big enough such that (1.1) possesses at least one positive solution.

In fact, for any $\lambda<\lambda_{1}$, there exists $b>0$ large enough such that for the function

$$
\left(\lambda_{1}-\lambda\right)-b \int_{\Omega}\left(\frac{1}{2} \varphi_{1}\right)^{r} d x+f\left(\frac{1}{2} \varphi_{1}\right)<0, \quad \forall x \in \Omega
$$

For above $b$, take $K>1+|\lambda|+\frac{1}{2}\left\|\varphi_{1}\right\|_{\infty}$ large enough such that

$$
f(K)>b K^{r}|\Omega|
$$

Let $\underline{u}=\frac{1}{2} \varphi_{1}(x)$ and $\bar{u}=K$. From (4.3) and (4.4), we have

(a) $\underline{u}=\frac{1}{2} \varphi_{1}<K=\bar{u}$ in $\Omega$ and $\underline{u}=\frac{1}{2} \varphi_{1}(x)=0<K=\bar{u}$ on $\partial \Omega$; 
(b)

$$
\begin{aligned}
-\Delta \underline{u}-\underline{u}\left(\lambda+b \int_{\Omega} u^{r} d x-f(\underline{u})\right) & =\frac{1}{2} \lambda_{1} \varphi_{1}-\frac{1}{2} \varphi_{1}\left(\lambda+b \int_{\Omega} u^{r} d x-f\left(\frac{1}{2} \varphi_{1}\right)\right) \\
& =\frac{1}{2} \varphi_{1}\left(\left(\lambda_{1}-\lambda\right)-b \int_{\Omega} u^{r} d x+f\left(\frac{1}{2} \varphi_{1}\right)\right) \\
& <0, \quad x \in \Omega, \forall u \in[\underline{u}, \bar{u}]
\end{aligned}
$$

and

$$
\begin{aligned}
-\Delta \bar{u}-\bar{u}\left(\lambda+b \int_{\Omega} u^{r} d x-f(\bar{u})\right) & =-0-K\left(\lambda+b \int_{\Omega} u^{r} d x-f(K)\right) \\
& =K\left(-\lambda-b \int_{\Omega} u^{r} d x+f(K)\right) \\
& \geq K\left(-\lambda-b K^{r}|\Omega|+f(K)\right) \\
& >0, \quad x \in \Omega, \forall u \in[\bar{u}, \bar{u}],
\end{aligned}
$$

which implies that $(\underline{u}, \bar{u})$ is a pair of sub-supersolutions to (1.1). Theorem 4.2 guarantees that (1.1) has at least one positive solution for all $\lambda<\lambda_{1}$.

The proof is complete.

Theorem 4.3 Assume that $b>0$ and $k_{n}<r$. There exists $\lambda^{*}>\lambda_{1}$ such that (1.1) possesses at least a positive solution if and only if $\lambda \leq \lambda^{*}$. Moreover,

$$
\lim _{b \rightarrow 0^{+}} \lambda^{*}(b)=+\infty \text { and } \lim _{b \rightarrow+\infty} \lambda^{*}(b)=\lambda_{1} .
$$

Proof Assume that $b>0$ and $k_{n}<r$. Define now

$$
\lambda^{*}=\sup \{\lambda \in \mathbb{R}:(1.1) \text { possesses at least a positive solution }\} .
$$

We know by Theorems 2.1 and 3.2 that $\lambda_{1}<\lambda^{*}<+\infty$.

Step 1. We prove now that there exists a positive solution for all $\lambda \in\left(-\infty, \lambda^{*}\right)$.

Indeed, take $\lambda \in\left[\lambda_{1}, \lambda^{*}\right)$, then there exists $\mu \in\left(\lambda_{1}, \lambda^{*}\right]$ such that (1.1) possesses at least a positive solution, denoted by $u_{\mu}$. Choose $\varepsilon$ small enough such that $\varepsilon \varphi_{1}<u_{\mu}$ for all $x \in \Omega$ such that

$$
f\left(\varepsilon \varphi_{1}\right)-b \int_{\Omega}\left(\varepsilon \varphi_{1}\right)^{r} d x \leq \lambda-\lambda_{1}
$$

Let $\underline{u}=\varepsilon \varphi_{1}$ and $\bar{u}=u_{\mu}$. Since $u_{\mu}$ is a positive solution of (1.1), from (4.5), one has

(a) $\underline{u}=\varepsilon \varphi_{1}<u_{\mu}$ in $\Omega$ and $\underline{u}=0=\bar{u}$ on $\partial \Omega$;

(b)

$$
\begin{aligned}
-\Delta \underline{u}-\underline{u}\left(\lambda+b \int_{\Omega} u^{r} d x-f(\underline{u})\right) & =\varepsilon \lambda_{1} \varphi_{1}-\varepsilon \varphi_{1}\left(\lambda+b \int_{\Omega} u^{r} d x-f\left(\varepsilon \varphi_{1}\right)\right) \\
& =\varphi_{1} \varepsilon\left(\left(\lambda_{1}-\lambda\right)-b \int_{\Omega} u^{r} d x+f\left(\varepsilon \varphi_{1}\right)\right) \\
& <0, \quad x \in \Omega, \forall u \in[\underline{u}, \bar{u}]
\end{aligned}
$$


and

$$
\begin{aligned}
&- \Delta \bar{u}-\bar{u}\left(\lambda+b \int_{\Omega} u^{r} d x-f(\bar{u})\right) \\
&=u_{\mu}\left(\mu+b \int_{\Omega} u_{\mu}^{r} d x-f\left(u_{\mu}\right)\right)-u_{\mu}\left(\lambda+b \int_{\Omega} u^{r} d x-f\left(u_{\mu}\right)\right) \\
&=u_{\mu}\left(\mu-\lambda+b \int_{\Omega}\left(u_{\mu}^{r}-u^{r}\right) d x\right) \\
&>0, \quad x \in \Omega, \forall u \in[\bar{u}, \bar{u}],
\end{aligned}
$$

which implies that $(\underline{u}, \bar{u})$ is a pair of sub-supersolutions to (1.1). Theorem 4.2 guarantees that (1.1) has at least one positive solution for all $\lambda \in\left[\lambda_{1}, \lambda_{*}\right)$.

Now, Theorem 2.1 implies $C_{0}$ is supercritical, which implies that there exists $(\lambda, u) \in C_{0}$ with $\lambda_{0}>\lambda_{1}$. For any $\lambda<\lambda_{1}$, let $K=\left[\lambda, \lambda_{0}\right]$. Theorem 3.2 guarantees that $\|u\|_{\infty} \leq L_{0}$ for all $\lambda \in K$, which together with the unboundedness of $C_{0}$ implies that there is $u$ such that $(\lambda, u) \in C_{0}$.

Step 2. We show that, for $\lambda=\lambda^{*},(1.1)$ has a positive solution.

Taking a sequence of positive solutions $\left(\lambda_{n}, u_{n}\right)$ of (1.1) such that $\lambda_{n} \leq \lambda^{*}$ and $\lambda_{n} \rightarrow \lambda^{*}$. Thanks to the bounds of Theorem 3.2, we have that $u_{n} \rightarrow u^{*} \geq 0, u^{*}$ is a solution for $\lambda=\lambda^{\star}$. Since $\lambda^{*}>\lambda_{1}$ and $\lambda_{1}$ is the unique bifurcation point from the trivial solution, we conclude that $u^{*}>0$.

Step 3. We show that

$$
\lim _{b \rightarrow 0^{+}} \lambda^{*}(b)=+\infty \text { and } \lim _{b \rightarrow+\infty} \lambda^{*}(b)=\lambda_{1}
$$

Observe that since $\lambda_{1}<\lambda^{*} \leq L_{1}$ defined in Theorem 3.2 and $\lim _{b \rightarrow \infty} L_{1}=\lambda_{1}$, we concluded that

$$
\lim _{b \rightarrow \infty} \lambda^{*}(b)=\lambda_{1}
$$

Now we prove

$$
\lim _{b \rightarrow 0^{+}} \lambda^{*}(b)=+\infty
$$

It suffices to show that, for any $\lambda>\lambda_{1}$, there exists $b>0$ small enough such that (1.1) possesses at least a positive solution. For $\lambda>\lambda_{1}$, take $\tilde{\Omega} \supset \Omega$ and consider $\tilde{\varphi}_{1}$ and $\tilde{\lambda}_{1}$ the positive eigenfunction and eigenvalue associated with $\tilde{\Omega}$. Choose $K$ large enough such that

$$
f\left(K \tilde{\varphi}_{1}(x)\right)-\lambda>0, \quad \forall x \in \bar{\Omega} .
$$

Choose $b>0$ small enough such that

$$
f\left(K \tilde{\varphi}_{1}(x)\right)-\lambda-b \int_{\Omega}\left(K \tilde{\varphi}_{1}\right)^{r} d x>0, \quad \forall x \in \bar{\Omega}
$$


Choose $\varepsilon>0$ small enough such that $\varepsilon \varphi_{1}<K \tilde{\varphi}_{1}$ and

$$
\lambda-\lambda_{1}+b \int_{\Omega}\left(\varepsilon \varphi_{1}\right)^{r} d x-f\left(\varepsilon \varphi_{1}\right)>0, \quad \forall x \in \Omega
$$

Let $\underline{u}(x)=\varepsilon \varphi_{1}(x)$ and $\bar{u}(x)=K \tilde{\varphi}_{1}(x)$ for $x \in \bar{\Omega}$. From (4.6) and (4.7), we have

(a) $\underline{u}=\varepsilon \varphi_{1}<K \tilde{\varphi}_{1}=\bar{u}(x)$ in $\Omega$ and $\underline{u}=0<K \tilde{\varphi}_{1}=\bar{u}$ on $\partial \Omega$;

(b)

$$
\begin{aligned}
-\Delta \underline{u}-\underline{u}\left(\lambda+b \int_{\Omega} u^{r} d x-f(\underline{u})\right) & =\varepsilon \lambda_{1} \varphi_{1}-\varepsilon \varphi_{1}\left(\lambda+b \int_{\Omega} u^{r} d x-f\left(\varepsilon \varphi_{1}\right)\right) \\
& =\varepsilon \varphi_{1}\left(\left(\lambda_{1}-\lambda\right)-b \int_{\Omega} u^{r} d x+f\left(\varepsilon \varphi_{1}\right)\right) \\
& <0, \quad x \in \Omega, \forall u \in[\underline{u}, \bar{u}]
\end{aligned}
$$

and

$$
\begin{aligned}
-\Delta \bar{u}-\bar{u}\left(\lambda+b \int_{\Omega} u^{r} d x-f(\bar{u})\right) & =K \tilde{\lambda} \tilde{\varphi}_{1}-K \tilde{\varphi}_{1}\left(\lambda+b \int_{\Omega} u^{r} d x-f\left(K \tilde{\varphi}_{1}\right)\right) \\
& =K \tilde{\varphi}_{1}\left(\tilde{\lambda}-\lambda-b \int_{\Omega} u^{r} d x+f\left(K \tilde{\varphi}_{1}\right)\right) \\
& >0, \quad x \in \Omega, \forall u \in[\bar{u}, \bar{u}],
\end{aligned}
$$

which implies that $(\underline{u}, \bar{u})$ is a pair of sub-supersolutions to (1.1). Theorem 4.2 guarantees that (1.1) has at least one positive solution for $\lambda>\lambda_{1}$.

The proof is complete.

Theorem 4.4 Assume that $b>0$ and $r=1<k_{n}$. There exists $\lambda_{*}<\lambda_{1}$ such that (1.1) possesses at least a positive solution if and only if $\lambda \geq \lambda_{*}$. Moreover,

$$
\lim _{b \rightarrow 0^{+}} \lambda_{*}(b)=\lambda_{1} \quad \text { and } \quad \lim _{b \rightarrow+\infty} \lambda_{*}(b)=-\infty .
$$

The proof of Theorem 4.4 is similar to that of Theorem 4.3, we omit it.

\section{Acknowledgements}

We are thankful to the referees for their valuable suggestions to improve this paper.

\section{Funding}

This work is supported by the National Natural Science Foundation of China (61603226) and the Fund of Natural Science of Shandong Province (ZR2018MA022).

Availability of data and materials

Not applicable.

\section{Competing interests}

The authors declare that they have no competing interests.

\section{Authors' contributions}

All authors contributed equally to the writing of this paper. All authors read and approved the final manuscript.

\section{Authors' information}

Not applicable 


\section{Author details}

${ }^{1}$ Life Science College, Shandong Normal University, Jinan, P.R. China. ${ }^{2}$ School of Mathematical Sciences, Shandong Normal University, Jinan, P.R. China.

\section{Publisher's Note}

Springer Nature remains neutral with regard to jurisdictional claims in published maps and institutional affiliations.

Received: 30 January 2018 Accepted: 13 September 2018 Published online: 27 September 2018

\section{References}

1. Ackleh, A.S., Ke, L.: Existence-uniqueness and long time behavior for a class of nonlocal nonlinear parabolic evolution equations. Proc. Am. Math. Soc. 128, 3483-3492 (2000)

2. Alves, C.O., Covei, D.P.: Existence of solution for a class of nonlocal elliptic problem via sub-supersolution method. Nonlinear Anal., Real World Appl. 23, 1-8 (2015)

3. Berestycki, H.: Le nombre de solutions de certains problèmes semi-linéaires elliptiques. J. Funct. Anal. 40(1), 1-29 (1981)

4. Cheng, H., Yuan, R.: Existence and stability of traveling waves for Leslie-Gower predator-prey system with nonlocal diffusion. Discrete Contin. Dyn. Syst., Ser. A 37, 5433-5454 (2017)

5. Chipot, M., Lovat, B.: Some remarks on nonlocal elliptic and parabolic problems. Nonlinear Anal. 30, 4619-4627 (1997)

6. Corrêa, F.J.S.A., Delgado, M., Suárez, A.: Some nonlinear heterogeneous problems with nonlocal reaction term. Adv. Differ. Equ. 16, 623-641 (2011)

7. Crandallm, M.G., Rabinowitz, P.H.: Bifurcation from simple eigenvalues. J. Funct. Anal. 8, 321-340 (1971)

8. Delgado, M., Figueiredo, G.M., Pimenta, M.T.O., Suárez, A.: Study of a logistic equation with local and nonlocal reaction terms. Topol. Methods Nonlinear Anal. 47(2), 693-713 (2016)

9. Delgado, M., López-Gómez, J., Suárez, A.: On the symbiotic Lotka-Volterra model with diffusion and transport effects. J. Differ. Equ. 160(1), 175-262 (2000)

10. Freitas, P.: Nonlocal reaction-diffusion equations, differential equations with applications to biology. Fields Inst. Commun. 21, 187-204 (1999)

11. Furter, J., Grinfeld, M.: Local vs. nonlocal interactions in population dynamics. J. Math. Biol. 27, 65-80 (1989)

12. Hsu, S.B., López-Gómez, J., Mei, L., Molina-Meyer, M.: A nonlocal problem from conservation biology. SIAM J. Math. Anal. 46, 435-459 (2014)

13. Li, X., Song, S.: Stabilization of delay systems: delay-dependent impulsive control. IEEE Trans. Autom. Control 62, 406-411 (2017)

14. Li, X., Wu, J.: Stability of nonlinear differential systems with state-dependent delayed impulses. Automatica 64, 63-69 (2016)

15. López-Gómez, J.: The maximum principle and the existence of principal eigenvalues for some linear weighted boundary value problems. J. Differ. Equ. 127, 263-294 (1996)

16. Mao, A., Zhang, Z.: Sign-changing and multiple solutions of Kirchhoff type problems without the P.S. condition Nonlinear Anal. 70, 1275-1287 (2009)

17. Protter, M.H., Weinberger, H.F.: Maximum Principles in Differential Equations. Springer, New York (1984)

18. Rabinowitz, P.H.: Some global results for nonlinear eigenvalue problems. J. Funct. Anal. 7(3), 487-513 (1971)

19. Shi, J., Yao, M.: On a singular nonlinear semilinear elliptic problem. Proc. R. Soc. Edinb. 12, 1389-1401 (1998)

20. Zhang, Z., Perera, K.: Sign changing solutions of Kirchhoff type problems via invariant sets of descent flow. J. Math. Anal. Appl. 317, 456-463 (2006)

\section{Submit your manuscript to a SpringerOpen ${ }^{\odot}$ journal and benefit from:}

- Convenient online submission

- Rigorous peer review

- Open access: articles freely available online

- High visibility within the field

- Retaining the copyright to your article

Submit your next manuscript at $\gg$ springeropen.com 